\title{
Estruturação de uma base de dados sobre a COVID-19 no estado do Rio Grande do Sul, Brasil
}

\author{
GASS, Sidnei Luís Bohn'; SILVA, Dieison Morozoli da²
}

1Professor da Universidade Federal do Pampa - Campus Itaqui, e do Programa de Pós-Graduação em Geografia da UFRGS. Coordenador do projeto SIGPampa - sidneigass@unipampa.edu.br http://orcid.org/0000-0001-5197-7506

2Discente do Curso de Engenharia Cartográfica e de Agrimensura da Universidade Federal do Pampa Campus Itaqui - dieison.ufp@gmail.com - http://lattes.cnpq.br/0116359981757404

\section{RESUMO}

O projeto SIGPampa, iniciado em 2017, vem desenvolvendo diversas atividades vinculadas ao uso das geotecnologias, em especial, àquelas baseadas em plataformas livres, como pode ser verificado no site do projeto em https://sites.unipampa.edu.br/sigpampa/

O
presente material tem o objetivo de apresentar a estruturação de uma base de dados referente a COVID-19, principalmente no que se refere a sua evolução no Rio Grande do Sul. O enfoque principal está baseado em duas linhas distintas de trabalho: a primeira, tomando por base todo o território do Rio Grande do Sul e, a segunda, com ênfase aos municípios com Campi da UNIPAMPA, para subsidiar pesquisas e atividades acadêmicas presentes e futuras. $O$ acesso aos materiais e informações produzidas estão disponíveis no endereço

https://sites.unipampa.edu.br/sigpampa/covid-19/. Caso queira contribuir com alguma sugestão para este material, a equipe do projeto está à disposição.

\begin{abstract}
The SIGPampa project, started in 2017, has been has been developing various activities related to the use of geotechnologies, especially those based on free platforms, as can be seen on the project's website

at

https://sites.unipampa.edu.br/sigpampa/ The purpose of this material is to present the structuring of a database referring to COVID-19, mainly with regard to its evolution in Rio Grande do Sul. The main focus is based on two distinct lines of work: the first, based on the entire territory of Rio Grande do Sul, and the second, with emphasis on the municipalities with UNIPAMPA campuses, to subsidize research and current and future academic activities. Access to the materials and information produced is available at https://sites.unipampa.edu.br/sigpampa/covid-19/.
\end{abstract} If you want to contribute with any suggestions for this material, the project team is available.

\footnotetext{
Primeira versão publicada em 02/04/2020.

Versão atualizada em 06/04/2020.

Todas as informações e produtos referentes a este trabalho podem ser acessadas no endereço https://sites.unipampa.edu.br/sigpampa/

Este é um trabalho em desenvolvimento, portanto, passará por atualizações e aprimoramentos.
} 


\section{INTRODUÇÃO}

Dentre as suas várias áreas de atuação, a Geografia também se ocupa da temática saúde. Os procedimentos de localização e representação de fenômenos para posterior modelagem e análise vem ganhando espaço nas mais variadas áreas do conhecimento. Tais procedimentos permitem uma melhor visualização e compreensão do comportamento dos fenômenos.

Após a deflagração da COVID-19, que é uma doença infecciosa causada pelo Coronavirus da Síndrome Respiratória Aguda Grave 2 (SARS-Cov-2) [1] [2] [3], várias iniciativas a nível mundial têm apresentado sistematizações de dados com o intuito de demonstrar sua evolução. A facilidade de acesso aos dados e as tecnologias para sua sistematização facilitam tais práticas.

O espaço geográfico sempre apresentou alguma relação com as eventualidades epidemiológicas. De fato, conforme Bonfim e Medeiros [4], já no século XIX foi demonstrado que a localização espacial dos casos de cólera estava relacionada com a localização de uma bomba pública de água e sendo assim o espaço foi um importante ponto para a inferências sobre o comportamento dos casos de doença.

Contudo, há que se tomar um especial cuidado com esta disseminação de dados, pois há várias iniciativas que tentam comparar a COVID-19 com outras pandemias ocorridas ao longo da história da humanidade, mas, em alguns casos, sem o devido cuidado de buscar um embasamento científico sobre o vírus que causa a doença e o seu comportamento. Neste sentido, cabe mencionar que são necessárias equipes multidisciplinares para um correto tratamento destes dados, para que se possa extrair informações consistentes e que auxiliem de forma efetiva na prevenção e no combate à doença.

O projeto SIGPampa, em desenvolvimento desde 2017 no Campus Itaqui da UNIPAMPA, hospedado no endereço eletrônico https://sites.unipampa.edu.br/sigpampa/, busca disseminar o uso de geotecnologias livres em diferentes áreas do conhecimento. Desde sua concepção já foram desenvolvidos diversos materiais didáticos, livros, artigos científicos entre outros trabalhos, sempre com o objetivo de aproximar a Universidade da sociedade.

Tomando por base a premissa central do projeto SIGPampa, o objetivo geral do presente material é apresentar a estruturação de uma base de dados sobre a COVID-19 no estado do Rio Grande do Sul. Cabe mencionar que, sob o aspecto analítico, serão apresentados poucos aprofundamentos, visto que para que se tenha coerência científica baseada no conhecimento sobre o vírus e a doença, serão necessários profissionais de outras áreas.

Assim sendo, serão apresentados mapas, gráficos e tabelas que demonstrarão a evolução da doença no Rio Grande do Sul, com o intuito de estruturar uma base de dados para futuras pesquisas que deverão buscar a associação com profissionais, em especial, da área da saúde, entre outras. Todos os materiais produzidos estarão disponíveis na forma de base de dados para uso aberto, visto que estão sendo elaborados a partir de dados púbicos, 
sendo necessária apenas a citação referente aos créditos das sistematizações apresentadas.

Visando contribuir com o monitoramento da COVID-19 no âmbito regional de inserção da UNIPAMPA, os dados também serão tratados considerando os municípios com Campi da Universidade, a saber: Alegrete, Bagé, Caçapava do Sul, Dom Pedrito, Itaqui, Jaguarão, Sant'Ana do Livramento, São Borja, São Gabriel e Uruguaiana, representados no mapa da figura 1.

Figura 1 - Localização dos municípios com Campi da UNIPAMPA

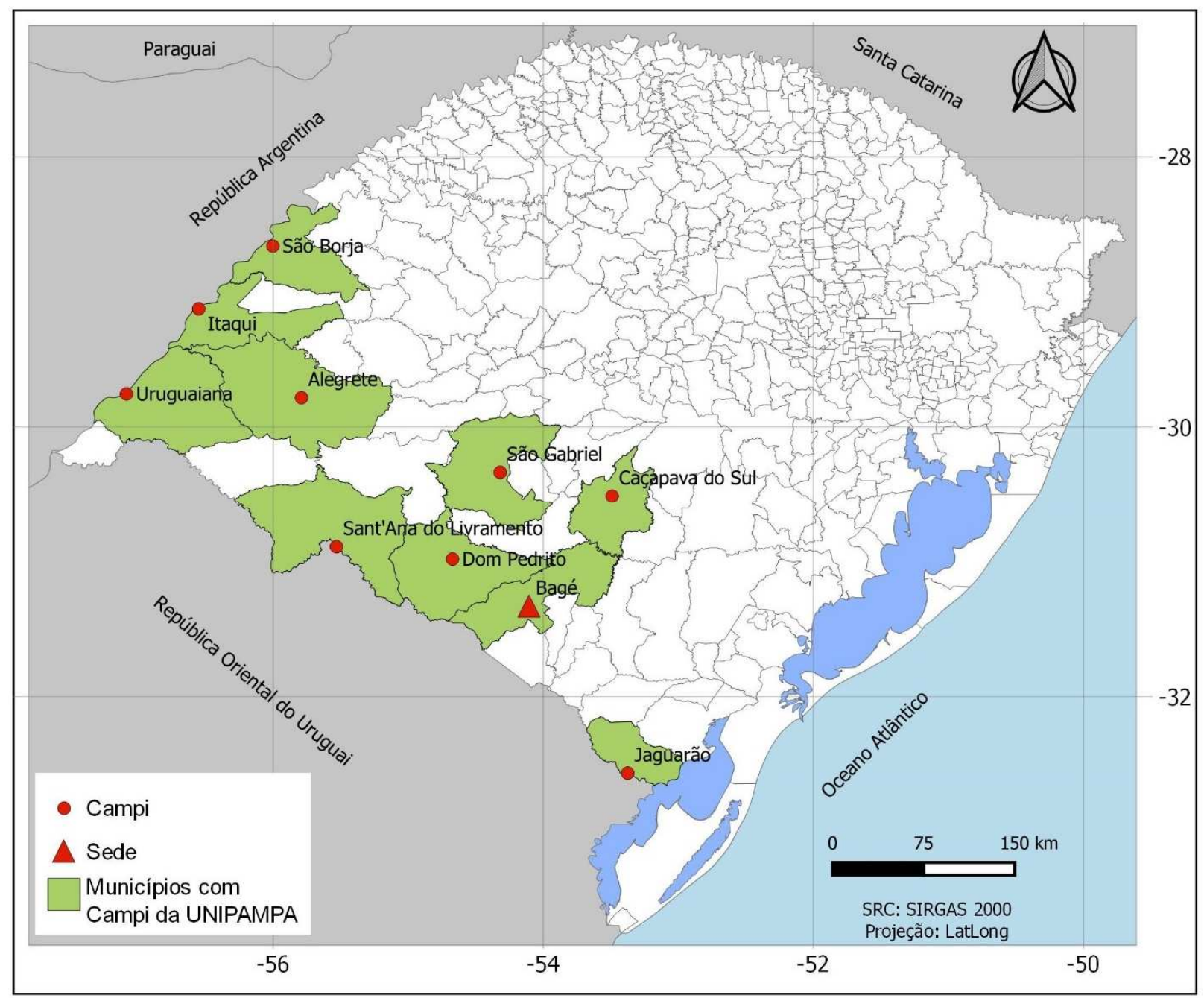

Fonte: elaborado pelos autores a partir de IBGE [8].

Cabe também a este material, instrumentalizar futuras aulas de Cartografia Temática, Sistemas de Informações Georreferenciadas e Geoprocessamento, pautadas em estudos de caso atuais e situações vivenciadas pelos docentes e discentes, além de ações de extensão universitária, servindo, assim, à tríade da ação universitária: ensino, pesquisa e extensão. 


\section{BREVE PERFIL POPULACIONAL DO RIO GRANDE DO SUL}

A compreensão da distribuição da população é um fator que pode auxiliar no entendimento de como os casos evoluem por faixa etária. De acordo com os dados do Censo Demográfico de 2010 [5], o Rio Grande do Sul contava com uma população de 10.693 .929 habitantes, dos quais $85,1 \%$ residiam nas áreas urbanas e $14,9 \%$ no meio rural. Ao compararmos estes dados com os do Censo Demográfico de 2000 (tabela 1) [6], será possível verificar que ocorre um processo de urbanização, com um aumento de $3,45 \%$ no período analisado.

Tabela 1 - Evolução da população do Rio Grande do Sul entre 2000 e 2010

\begin{tabular}{l|r|r|r|r}
\hline \multirow{2}{*}{ Variável } & \multicolumn{2}{|c|}{ Ano de 2000 } & \multicolumn{2}{c}{ Ano de 2010 } \\
\cline { 2 - 5 } & Habitantes & \% & Habitantes & $\%$ \\
\hline Total & $\mathbf{1 0 1 8 7 7 9 8}$ & & $\mathbf{1 0 6 9 3 9 2 9}$ & \\
\hline Homens & 4994719 & 49.03 & 5205057 & 48.67 \\
\hline Mulheres & 5193079 & 50.97 & 5488872 & 51.33 \\
\hline Urbana & $\mathbf{8 3 1 7 9 8 4}$ & $\mathbf{8 1 . 6 5}$ & $\mathbf{9 1 0 0 2 9 1}$ & $\mathbf{8 5 . 1 0}$ \\
\hline Homens & 4018384 & 48.31 & 4370784 & 48.03 \\
\hline Mulheres & 4299600 & 51.69 & 4729507 & 51.97 \\
\hline Rural & $\mathbf{1 8 6 9 8 1 4}$ & $\mathbf{1 8 . 3 5}$ & $\mathbf{1 5 9 3 6 3 8}$ & $\mathbf{1 4 . 9 0}$ \\
\hline Homens & 976335 & 52.22 & 834273 & 52.35 \\
\hline Mulheres & 893479 & 47.78 & 759365 & 47.65 \\
\hline
\end{tabular}

Fonte: IBGE, Censos Demográficos de 2000 e 2010. Elaborado pelos autores.

Ao estratificar a população total do Rio Grande do Sul, para o ano de 2010, em faixas etárias (figura 2), é possível perceber que a maior concentração da população $(57,31 \%)$ se encontra na faixa etária de 20 a 59 anos.

Na pirâmide etária da figura 3, é possível observar um comparativo entre a população total do estado e os casos confirmados para COVID-19 até o dia 29/03/2020. Em primeira análise, percebe-se que o maior número de casos confirmados ocorre nas faixas de 30 a 39 e de 50 a 59 anos $(40,58 \%$ dos casos), sendo que nestas mesmas faixas etárias a população equivale a $26,27 \%$ do total, ocorrendo um comportamento diferenciado entre a população total e os casos confirmados, como também já mencionaram Dagnino, Weber e Panitz [7]. Portanto, este é um fenômeno que merece destaque em pesquisas especializadas futuras. Cabe também destacar que, com a evolução do número de casos confirmados, o comportamento da pirâmide etária poderá se alterar, fato que deverá ser acompanhado a partir da sistematização dos dados objeto deste material. 
Figura 2 - Distribuição da população total, urbana e rural do Rio Grande do Sul, por faixas etárias, para o ano de 2010

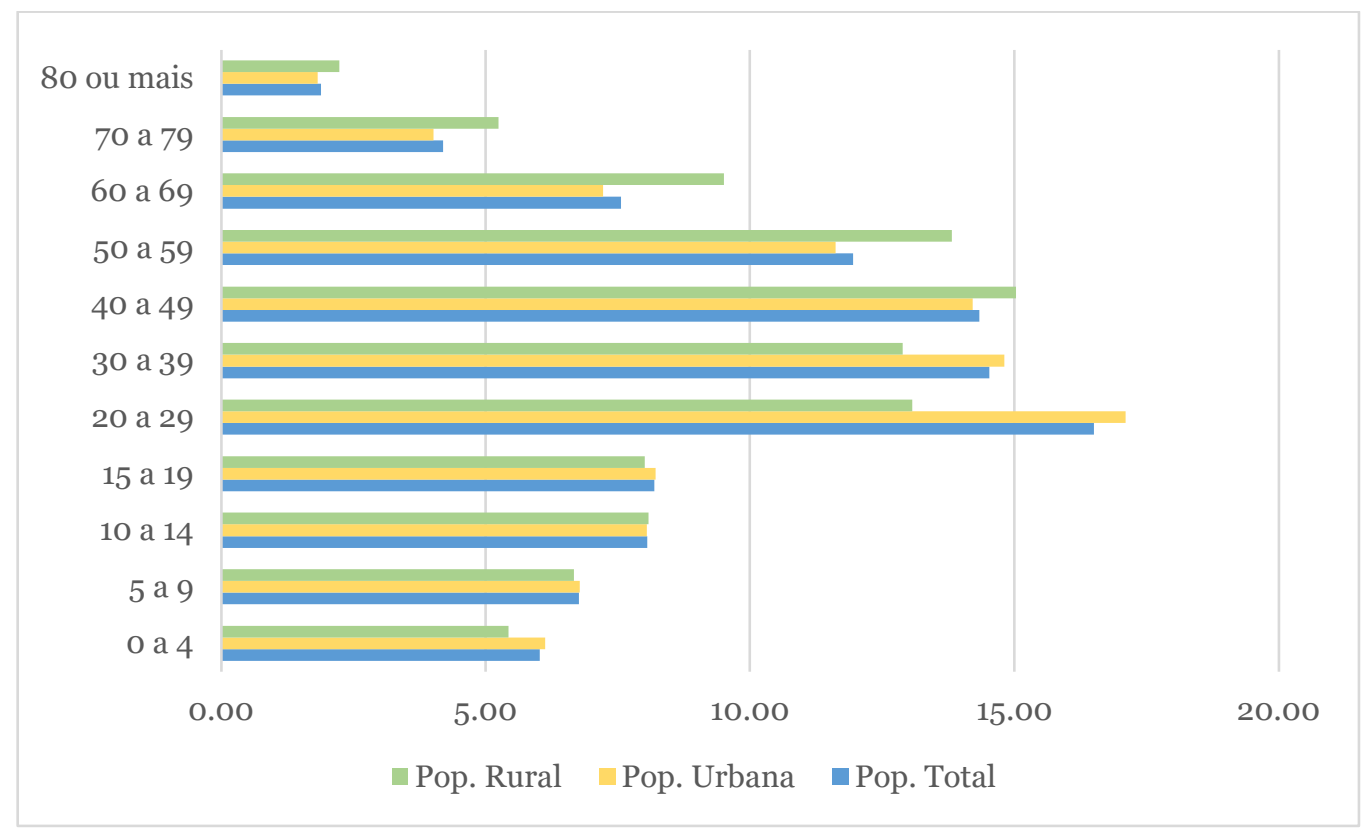

Fonte: IBGE, Censos Demográficos de 2000 e 2010. Elaborado pelos autores.

Figura 3 - Distribuição, por faixa etária, da população total para o ano de 2010, e dos casos confirmados para COVID-19 até 29/03/2020

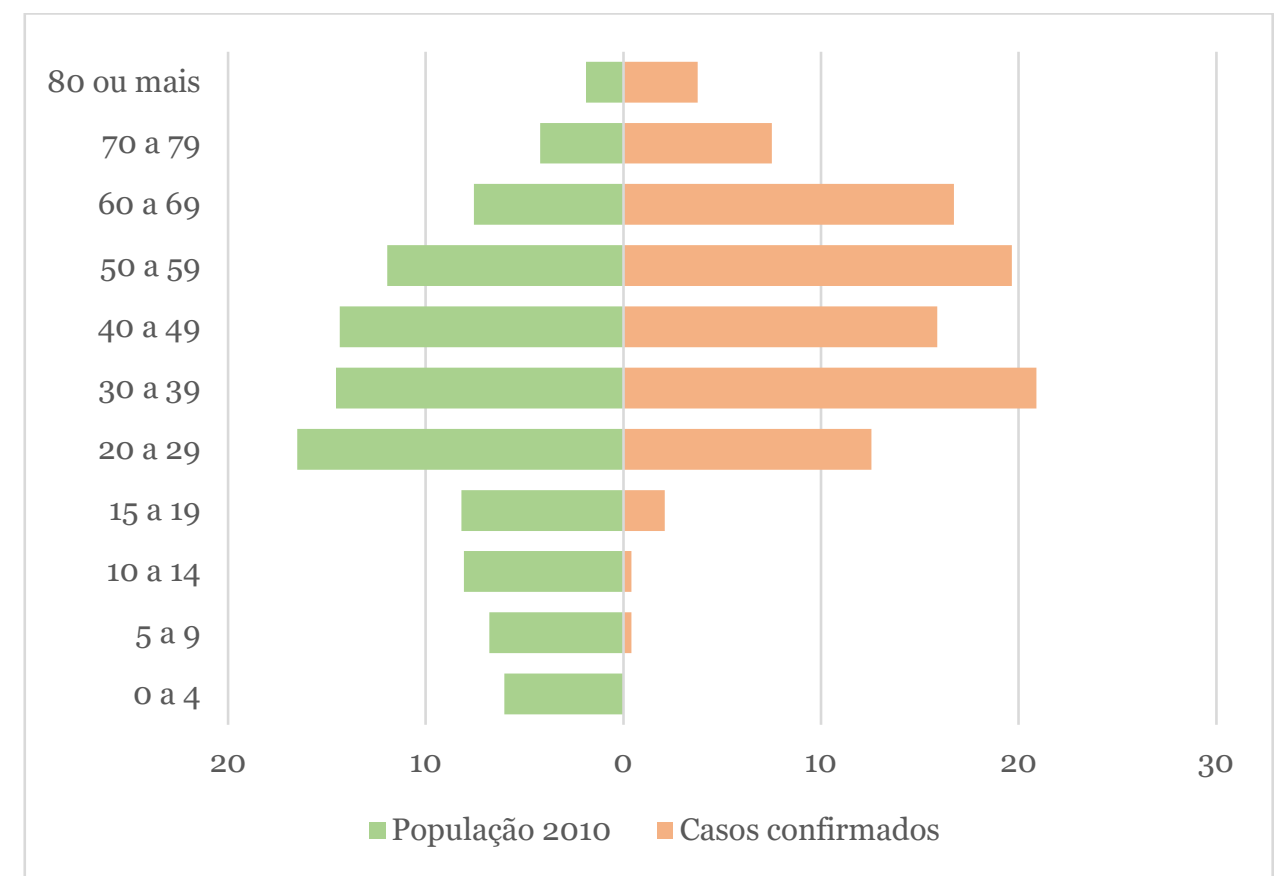

Os intervalos das faixas etárias utilizadas neste gráfico, coincidem com os intervalos do painel de dados da Secretaria Estadual da Saúde.

Fonte: IBGE, Censo Demográfico 2010 e painel de dados da Secretaria Estadual da Saúde. Elaborado pelos autores. 


\section{A DISTRIBUIÇÃO DOS CASOS PARA O RIO GRANDE DO SUL ATÉ O DIA 01/04/2020}

Até o dia 01/04/2020, o Rio Grande do Sul contava com 316 casos confirmados (figura 4), distribuídos em 53 municípios. Como pode ser observado no mapa da figura 5, majoritariamente os casos estão concentrados na região metropolitana de Porto Alegre, no litoral norte e na região de Bagé.

Figura 4 - Curva de evolução do número de casos confirmados para COVID-19 no Rio Grande do Sul, até o dia 01/04/2020

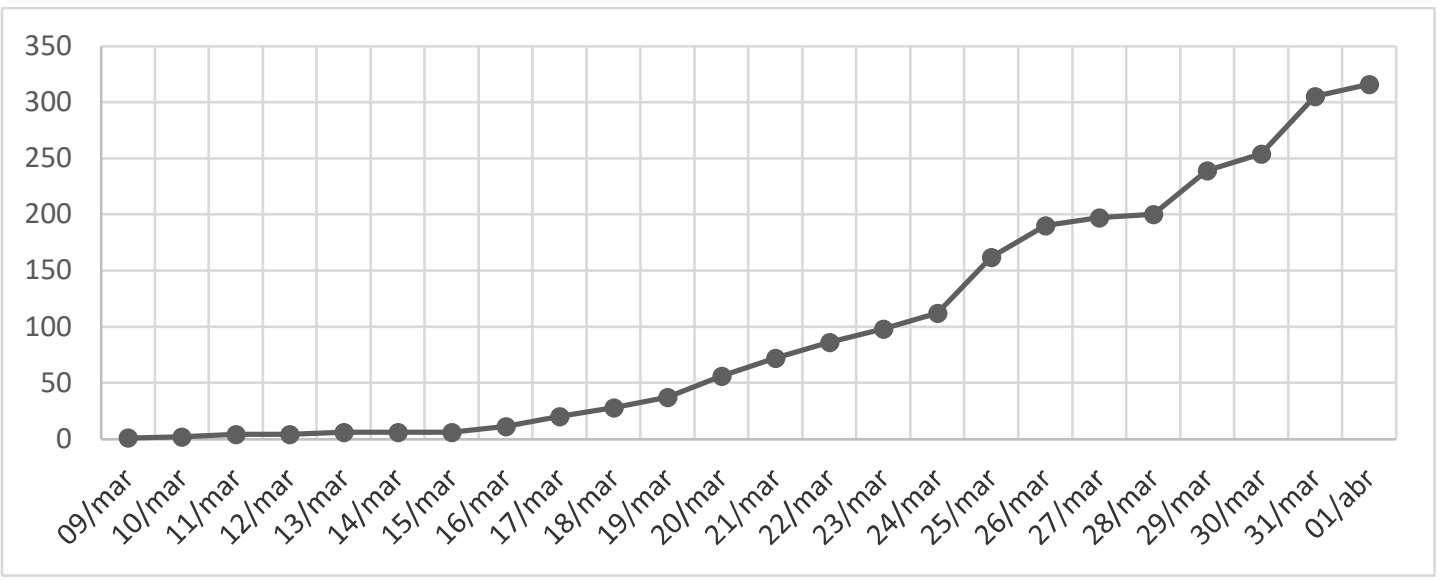

Fonte: painel de dados da Secretaria Estadual da Saúde. Elaborado pelos autores.

Figura 5 - Mapa de distribuição dos casos confirmados para COVID-19 no Rio Grande do Sul, até o dia 01/04/2020

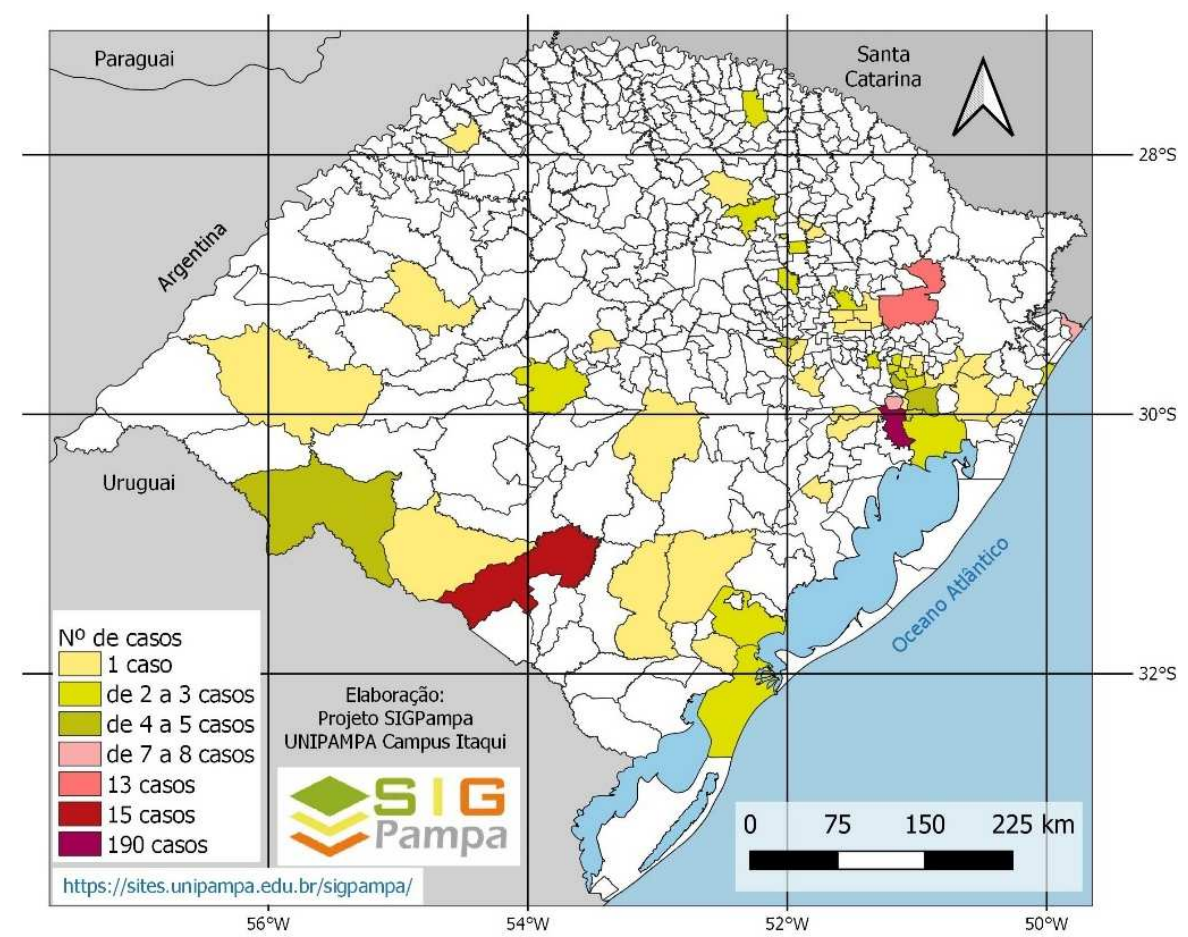

Fonte: Dados do painel de acompanhamento dos casos da Secretaria Estadual da Saúde do estado do Rio Grande do Sul. Elaborado pelos autores. 


\section{A DISTRIBUIÇÃO DOS CASOS PARA OS MUNICÍPIOS COM CAMPI DA UNIPAMPA ATÉ O DIA 01/04/2020}

Até o dia 01/04/2020, os municípios de atuação da UNIPAMPA, contavam com 22 casos confirmados (figura 6), distribuídos em 4 municípios (Alegrete, Bagé, Dom Pedrito e Sant'Ana do Livramento). Para o acompanhamento da evolução dos casos nos municípios de atuação da UNIPAMPA, foi criada uma plataforma on-line (figura 7) que pode ser acessada através do endereço https://qgiscloud.com/sigpampa/COVID 19 UNIPAMPA.

Figura 6 - Curva de evolução do número de casos confirmados para COVID-19 nos municípios com Campi da UNIPAMPA, até o dia 01/04/2020

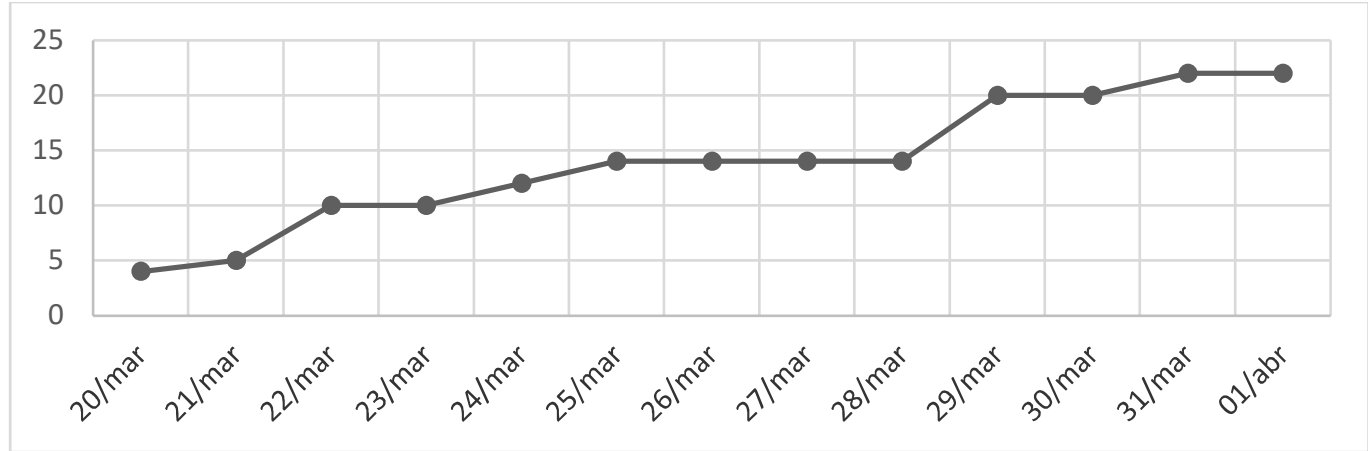

Fonte: painel de dados da Secretaria Estadual da Saúde. Elaborado pelos autores.

Figura 7 - Tela da plataforma desenvolvida para o acompanhamento da evolução dos casos nos municípios com Campi da UNIPAMPA

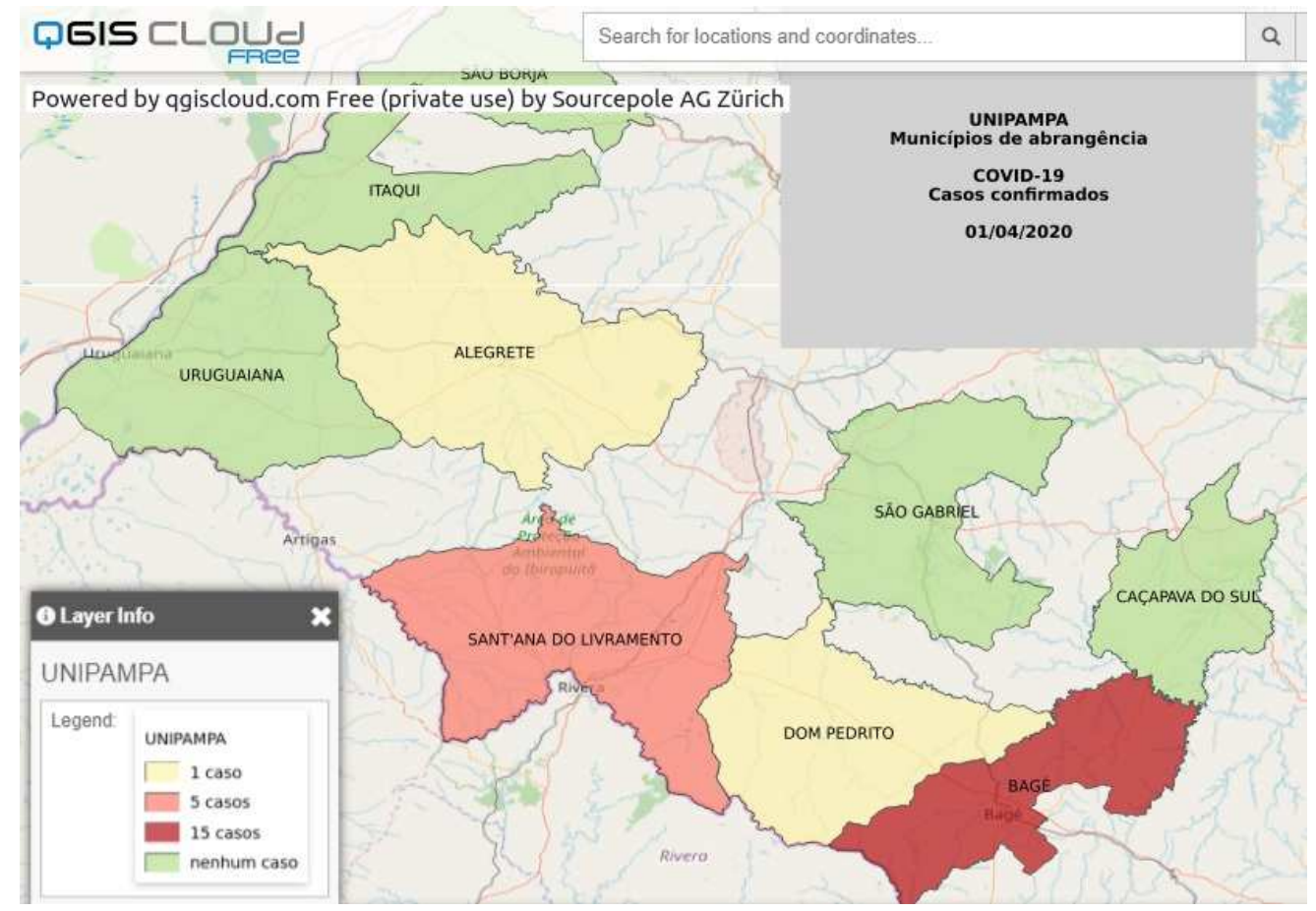

Fonte: Dados do painel de acompanhamento dos casos da Secretaria Estadual da Saúde do estado do Rio Grande do Sul. Elaborado pelos autores. 


\section{MATERIAIS E MÉTODOS}

Para o desenvolvimento da presente pesquisa estão sendo utilizadas planilhas de cálculo eletrônico para a elaboração das tabelas e gráficos e, para a elaboração dos mapas, está sendo utilizada a plataforma QGIS 3.10.3, que é a principal ferramenta de trabalho do projeto SIGPampa. Para a criação da plataforma on-line de acompanhamento dos casos nos municípios em que a UNIPAMPA possui Campi, está sendo utilizado o plugin QGISCloud, que permite a publicação dos dados através de Web Map Service.

Os dados que abastecem a base em construção são oriundos das seguintes fontes:

Dados populacionais dos Censos Demográficos dos anos de 2000 e 2010, sistematizados a partir da tabela 1552 - População residente, por situação do domicílio e sexo, segundo a forma de declaração da idade e a idade [5] [6];

Número de casos confirmados para COVID-19, sistematizados através dos informes epidemiológicos da Secretaria Estadual da Saúde do Rio Grande do Sul, bem como a partir da plataforma de disponibilização de informações, acessível pelo endereço http://ti.saude.rs.gov.br/covid19/;

Malha Territorial Municipal, inserida na Base Cartográfica Vetorial Contínua em escala 1:250.000, em sua versão 2017 [8].

Para a estruturação da base de dados, são executados os seguintes procedimentos:

Organização dos dados referentes aos casos em planilha eletrônica e posterior inserção em tabela de atributos do arquivo no formato Shape que contém os limites dos municípios do Rio Grande do Sul;

Análise da distribuição dos casos para a geração dos mapas temáticos. O método cartográfico que vem sendo aplicado é o coroplético, por representar melhor a distribuição dos dados;

Construção da curva de evolução dos casos, por data de confirmação e por data de coleta da amostra;

Disponibilização dos mapas e demais dados na página específica para esta temática, hospedada no endereço https://sites.unipampa.edu.br/sigpampa/covid-19/. Optouse em disponibilizar os mapas estáticos (em formato JPEG) para que os usuários possam ter a sua disposição um documento cartográfico padronizado e que possa ser utilizado em diferentes plataformas, desde que mantidos os seus créditos.

\section{DIVULGAÇÃO DOS DADOS COMPILADOS}

A cada nova compilação, os dados são disponibilizados na página específica para esta temática, hospedada no endereço https://sites.unipampa.edu.br/sigpampa/covid-19/. De forma sistemática, a página passa por atualizações, podendo ocorrer alterações na forma de disponibilização. 
Os arquivos que vem sendo disponibilizados são:

Mapa coroplético com a distribuição de casos por município, no formato JPEG;

Planilha atualizada com a evolução do número de casos por data e município, em formato PDF;

Curva da evolução dos casos por data de informação e dará da coleta das amostras, em formato PDF;

Óbitos por data e município, em formato PDF;

Pirâmide comparativa entre a distribuição da população de 2010 e os casos confirmados, por faixa etária, em formato PDF.

Está em processo de análise e sistematização, a disponibilização dos dados em formatos editáveis (xlsx, shp etc), para que o usuário possa fazer uso deles em suas próprias plataformas de trabalho.

\section{OUTRAS PESQUISAS EM ANDAMENTO}

Como já mencionado na introdução do presente material, há várias pesquisas com focos distintos, e outras que convergem entre si, em andamento a nível estadual, nacional e mundial. Neste sentido, mencionamos aqui àquelas que se vinculam de forma direta ao estado do Rio Grande do Sul, como pode ser verificado no quadro 1.

Quadro 1 - Outras pesquisas em desenvolvimento

\begin{tabular}{|c|c|}
\hline Descrição & Acesso \\
\hline $\begin{array}{l}\text { Pesquisa coordenada pela UFPel estará } \\
\text { baseada em amostragens epidemiológicas } \\
\text { sequenciais }\end{array}$ & $\begin{array}{l}\text { https://saude.rs.gov.br/rio-grande-do-sul-tera- } \\
\text { pesquisa-inedita-sobre-avanco-do-coronavirus }\end{array}$ \\
\hline $\begin{array}{l}\text { Pesquisa em desenvolvimento pelo projeto } \\
\text { SIG Litoral, do Campus Litoral Norte, da } \\
\text { UFRGS [7] [9] }\end{array}$ & https://www.ufrgs.br/sig/mapas/covid19-rs/ \\
\hline $\begin{array}{l}\text { Estimativa temporal dos casos para o Brasil } \\
\text { e o Rio Grande do Sul, elaborada pelos } \\
\text { pesquisadores do Laboratório GDISPEN, da } \\
\text { Universidade Federal de Pelotas }\end{array}$ & 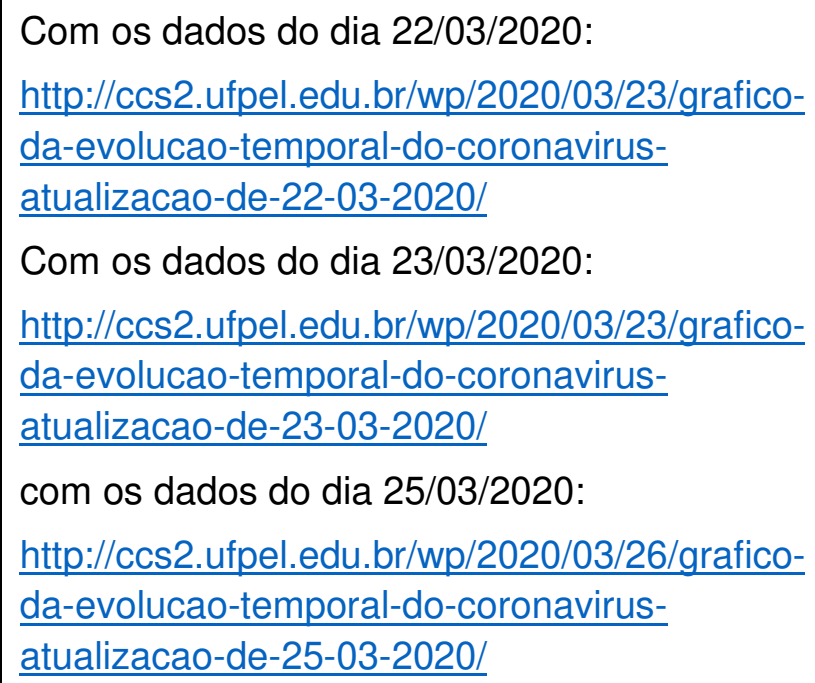 \\
\hline
\end{tabular}




\begin{tabular}{|c|c|}
\hline & 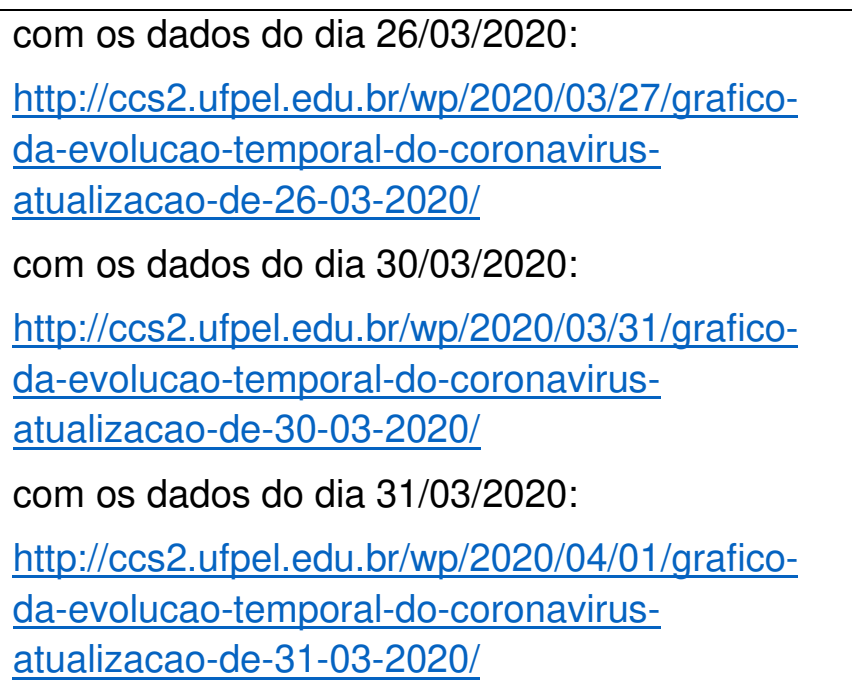 \\
\hline $\begin{array}{l}\text { Projeção de cenários para o Coronavirus } \\
\text { elaborados pela Secretaria de Planejamento, } \\
\text { Orçamento e Gestão }\end{array}$ & https://planejamento.rs.gov.br/cenariocoronavirus \\
\hline
\end{tabular}

Fonte: elaborado pelos autores.

\section{CONSIDERAÇÕES FINAIS}

O papel das Instituições Federais de Ensino Superior, em momentos de crise como o que se instalou a nível mundial, é potencializar os seus recursos humanos para que possam auxiliar, a partir das suas áreas de conhecimento, no desenvolvimento de pesquisas e ações que, de alguma forma, auxiliem na mitigação dos efeitos negativos que vem sendo sofridos. Esta é uma das premissas que fundamentam o presente material. Assim, a equipe do projeto SIGPampa espera poder dar a sua contribuição científica neste momento, através dos materiais aqui apresentados e das pesquisas em desenvolvimento, que deverão se vincular aos três pilares de sustentação da universidade, à saber: o ensino, a pesquisa e a extensão.

\section{REFERÊNCIAS BIBLIOGRÁFICAS}

[1] Wikipedia. Coronavirus. https://pt.wikipedia.org/wiki/Coronav\%C3\%ADrus. Acessado em 30 de mar. 2020.

[2] Wikipedia. COVID-19. https://pt.wikipedia.org/wiki/COVID-19. Acessado em 30 de mar. 2020.

[3] Wikipedia. Pandemia de COVID-19. https://pt.wikipedia.org/wiki/Pandemia de COVID-19. Acessado em 30 de mar. 2020.

[4] Bonfim, C.; Medeiros, Z. Epidemiologia e geografia: dos primórdios ao geoprocessamento. Revista Espaço para a Saúde, Londrina, v. 10, n. 1, p. 53-62, dez. 2008.

[5] IBGE. Censo 2010. Tabela 1552 - População residente, por situação do domićílio e sexo, segundo a forma de declaração da idade e a idade. Disponível em https://sidra.ibge.gov.br/tabela/1552. Acessado em 30 de mar. 2020.

[6] IBGE. Censo 2000. Tabela 1552 - População residente, por situação do domicílio e sexo, segundo a forma de declaração da idade e a idade. Disponível em https://sidra.ibge.gov.br/tabela/1552. Acessado em 30 de mar. 2020. 
[7] Dagnino, R.; Weber, E. J.; Panitz, L. M. Monitoramento do Coronavírus (Covid-19) nos municípios do Rio Grande do Sul, Brasil. SocArXiv, 28 Mar. 2020. https://doi.org/10.31235/osf.io/3uqn5

[8] IBGE. Base cartográfica vetorial contínua do Brasil em escala 1:250.000, versão 2017. Disponível em http://geoftp.ibge.gov.br/cartas e mapas/bases cartograficas continuas/bc250/versa02017/

[9] Dagnino, R.; Weber, E.; Panitz, L. Coronavírus (Covid-19) nos municípios do Rio Grande do Sul. Harvard Dataverse, V1, 28 mar. 2020. https://doi.org/10.7910/DVN/JK4STL 